\title{
Effect of annealing on the giant Hall effect
}

\author{
X. N. Jing, ${ }^{*}$ N. Wang, A. B. Pakhomov ${ }^{\dagger}$ K. K. Fung, and X. Yan \\ Department of Physics, Hong Kong University of Science of Technology, Clear Water Bay, Kowloon, Hong Kong
}

(Received 31 January 1996)

\begin{abstract}
We studied resistivity, magnetoresistivity, and Hall resistivity for a few percolating granular $\mathrm{NiFe}-\mathrm{SiO}_{2}$ films upon thermal annealing. It was found that both the resistivity and the Hall resistivity decrease by two orders of magnitude upon annealing at $520^{\circ} \mathrm{C}$, accompanied by a crossover from an approximately $-\ln T$ dependence of resistivity to a metallic behavior. On the other hand, transmission electron microscopy study showed that this pattern of resistivity and Hall resistivity change is not due to any new phases, but is associated with changes in particle size distribution. These results cannot be understood in the existing theoretical framework. [S0163-1829(96)06621-0]
\end{abstract}

Magnetic granular thin films, consisting of nanometer scale ferromagnetic metal particles embedded in a nearly immiscible insulating or metallic medium, have attracted much attention in the last decades ${ }^{1,2}$ and continue to be an active research area. Recent interest is due to the discovery of giant magnetoresistance in magnetic-nonmagnetic binary metal granular films. ${ }^{3,4}$ For metal-insulator films, it is known that a number of properties, such as resistivity, increase sharply when the metal volume fraction $x$ approaches $x_{c}$, the critical value for the metal-insulator transition. Recent studies of magnetic and magnetotransport properties in cosputtered $(\mathrm{NiFe})_{x}\left(\mathrm{SiO}_{2}\right)_{1-x}$ films showed that the extraordinary Hall resistivity can be enhanced by four orders of magnitude, compared to that of the continuous magnetic metal films, when $x \rightarrow x_{c}\left(x_{c} \approx 0.53\right)$ from above. 5,6 The enhancement factor of $10^{4}$ is considerably greater than the factor of $\sim(t / \Phi)^{g / \nu} \leqslant 30$ [for film thickness $t$ of $1 \mu \mathrm{m}$, and granular (particle) size $\Phi \sim 1 \mathrm{~nm}]$, expected from the conventional percolation model, where $g(\sim 0.4)$ and $\nu(\sim 0.9)$ are the $3 \mathrm{~d}$ percolation critical indices of the Hall resistivity and of the correlation length. ${ }^{7}$ This effect, which was termed the "giant Hall effect' by analogy with the giant magnetoresistance, was suggested as a basis for alternative candidate materials structure for magnetic-field sensors. ${ }^{5,6}$ In this work, resistivity, magnetoresistivity, Hall resistivity, and magnetization were studied for a few percolating granular $\mathrm{NiFe}-\mathrm{SiO}_{2}$ films with different microstructures altered by thermal annealing. It was found that both the resistivity and the Hall resistivity increase upon annealing at temperatures up to $300^{\circ} \mathrm{C}$, and decrease by two or more orders of magnitude upon annealing at $520^{\circ} \mathrm{C}$. Transmission electron microscopy (TEM) studies showed that this pattern of Hall resistivity change was not due to any new phases, but rather due to the changes in particle size distribution that may be associated with the connectivity of the metal granules.

Percolating granular $(\mathrm{Ni}-\mathrm{Fe})_{x}\left(\mathrm{SiO}_{2}\right)_{1-x}$ films of $1 \mu \mathrm{m}$ thick were prepared using a magnetron rf cosputtering system as described elsewhere, ${ }^{8}$ the substrates being held at $150{ }^{\circ} \mathrm{C}$ during sputtering. The metal volume fraction $x$ and the relative $\mathrm{Fe}$ content in $\mathrm{NiFe}$ were determined by energydispersive $\mathrm{x}$-ray (EDX) spectroscopy using a Philips EDAX XL30, to be $0.58,0.60$, and 0.63 , and $0.15,0.14$, and 0.14 , respectively, for three of the samples studies. ${ }^{5,6} \mathrm{We}$ will mainly discuss the results for the system with $x=0.58$; other samples behave in a qualitatively similar way, though the values of the quantities are different. For annealing, samples were placed in a quartz tube that was evacuated for half an hour and purged six times using high-purity helium gas. Before annealing, the quartz tube was filled with about 0.8 atm helium gas and was kept at that pressure throughout the annealing process. The quartz tube was inserted into the furnace at the preset temperature, and was pulled out to quench to room temperature after annealing for one hour.

The Hall resistance was measured using a five-terminal method with a potentiometer. The potentiometer was adjusted so that zero Hall voltage was detected in zero field. Care was taken to ensure that the positions of the electric contacts remained the same after subsequent annealing, to eliminate geometric error. Magnetization was measured using a Quantum Design superconducting quantum interference device magnetometer. For all field-dependent measurements, magnetic field was applied perpendicular to the film plane. For the TEM study, separate samples (as-deposited, and annealed at 300 and at $520^{\circ} \mathrm{C}$ ) were prepared in plane view for comparison. First, square pieces $(\sim 2.5 \times 2.5$ $\mathrm{mm}^{2}$ ) of the samples were cleaved from the material and glued onto TEM hole grids. The samples were mechanically polished from the back to reduce the thickness down to 10-30 $\mu \mathrm{m}$, and then each sample was thinned by argon ion milling to about $10-40 \mathrm{~nm}$. To avoid heating the samples during ion milling, the sample holder was cooled by liquid nitrogen. TEM images were taken using a Philips CM-20 electron microscope at $200 \mathrm{kV}$.

In bulk ferromagnetic metals, the Hall resistivity $\rho_{x y}$ consists of two terms, of which the ordinary Hall resistivity, due to the Lorentz force, is proportional to the field. The second term, the extraordinary part, is due to scattering of the polarized conduction $d$ electrons, and hence is proportional to magnetization. ${ }^{9}$ If the extraordinary Hall coefficient is much greater than the ordinary one, the plots of Hall resistivity against field will be similar to the magnetization curves, but with a finite slope at fields above saturation. Figure 1 shows a typical Hall resistivity as a function of the magnetic field $H$ measured at $T=5 \mathrm{~K}$ in the sample with $x=0.58$. The curve has a shape qualitatively similar to that in bulk metals. ${ }^{5,6,10}$ The Hall resistivities are negative in the positive field for all samples studied. 


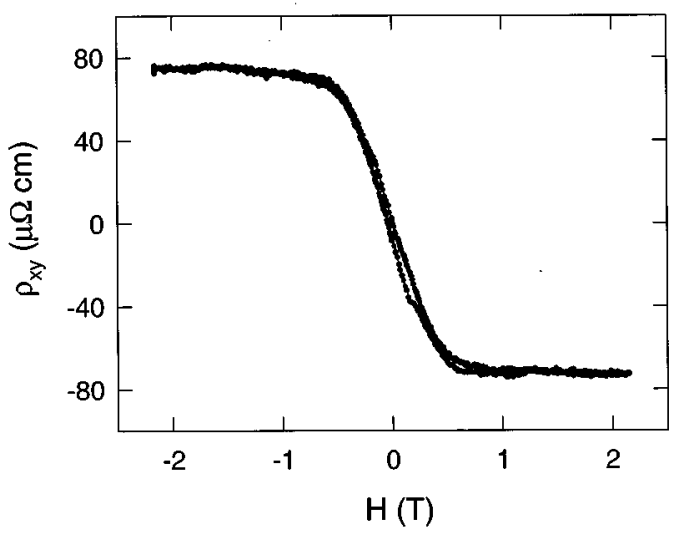

FIG. 1. Hall resistivity $\rho_{x y}$ as a function of magnetic field for an as-deposited granular film with the metal volume fraction $x=0.58$ at $T=5 \mathrm{~K}$.

Figure 2 shows the resistivity $\rho_{x x}$, the absolute values of saturation Hall resistivity $\rho_{x y s}$, the magnetoresistivity at $H=1.5 \mathrm{~T}$, or $\Delta \rho_{x x}=\rho_{x x}(0)-\rho_{x x}(1.5 \mathrm{~T})$, and the saturation magnetization $M_{s}$, all measured at $5 \mathrm{~K}$, as functions of the annealing temperature $T_{a}$. In this work, both the saturation magnetization and the Hall resistivity were determined at $H=1.5 \mathrm{~T}$. For $T_{a} \leqslant 300{ }^{\circ} \mathrm{C}, \rho_{x x}, \Delta \rho_{x x}$, and $\rho_{x y s}$ increase monotonically with increasing annealing temperature. The total enhancement of $\rho_{x x}$ is about $50 \%$ and that of $\rho_{x y s}$ is about $70 \%$ after annealing at $300{ }^{\circ} \mathrm{C}$ compared to the values for the as-deposited film, while $M_{s}$ remains nearly constant or decreases slightly $(<7 \%)$. Upon further annealing above $420^{\circ} \mathrm{C}$, however, $\rho_{x x}, \Delta \rho_{x x}$, and $\rho_{x y s}$ decreased considerably (by a factor of $\geqslant 10^{2}$ ), while the saturation magnetization $M_{s}$ increased by $\sim 20 \%$. Similar behavior of $\rho_{x x}$, $\Delta \rho_{x x}, \rho_{x y}$, and $M_{s}$ was observed for samples with $x=0.60$ and 0.63 .

Figure 3 shows normalized resistivity at zero field as a function of measurement temperature, where the temperature scale is logarithmic, for samples annealed at different temperatures. The temperature coefficient of resistivity (TCR) is negative at low temperatures for all samples. Remarkably,
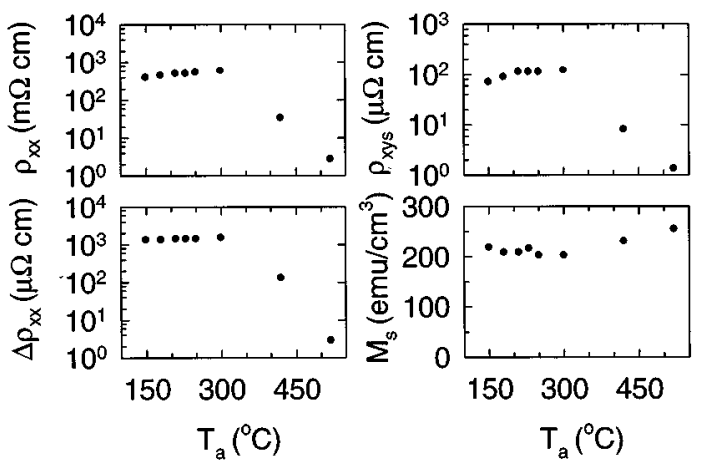

FIG. 2. Resistivity $\rho_{x x}$, magnetoresistivity $\Delta \rho_{x x}$, saturation Hall resistivity $\rho_{x y s}$, and saturation magnetization $M_{s}$ as functions of the annealing temperature $T_{a}$, for samples with $x=0.58$. The measurement temperature is $5 \mathrm{~K}$. For the as-deposited sample, $T_{a}=150{ }^{\circ} \mathrm{C}$ is the substrate temperature during deposition.

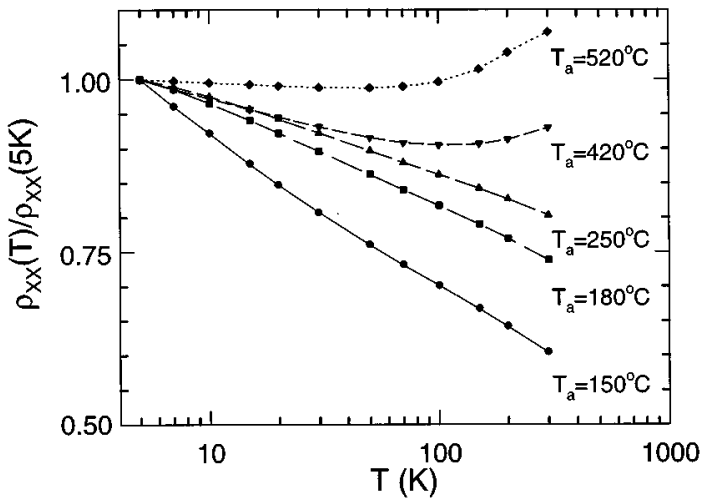

FIG. 3. Normalized resistivity at zero field as a function of measurement temperature for samples with $x=0.58$ annealed at different temperatures $T_{a}$.

for samples annealed at low temperatures it is negative even at room temperature. The temperature dependences of resistivity are close to logarithmic, with the slope decreasing with increasing annealing temperature. Comparing the results in Figs. 2 and 3, it can be concluded that the giant Hall resistivity is associated with a large resistivity with a $-\ln T$ temperature dependence. Also the increase in the giant Hall resistivity upon annealing is associated with an increase in the resistivity and a decrease in the slope $-d \rho_{x x} / d(\ln T) /$ $\rho_{x x}(5 \mathrm{~K})$. Upon high-temperature annealing $(420$ and $520^{\circ} \mathrm{C}$ ), the giant Hall effect disappears, accompanied by the change from a large resistivity with $-\ln T$ dependence to a small metalliclike resistivity. Note that the value of $\rho_{x y s} \sim 1 \mu \Omega \mathrm{cm}$ upon annealing at $520{ }^{\circ} \mathrm{C}$ is close to what can be expected from the conventional percolation theory at the percolation threshold $x_{c}$, or $\rho_{x y s}\left(x_{c}\right)$ $\sim \rho_{x y s}(1)(t / \Phi)^{g / \nu},{ }^{11}$ where $\rho_{x y s}(1) \sim 3.7 \times 10^{-2} \mu \Omega \mathrm{cm}$ (Ref. 5) is the Hall resistivity for $x=1$.

Figure 4 shows the dark-field TEM images for the samples with $x=0.58$ as deposited (a), annealed at $300{ }^{\circ} \mathrm{C}$ (b), and at $520^{\circ} \mathrm{C}$ (c). Insets show selected area electron diffraction (SAD) patterns. The structure of the particles in the granular films was identified by SAD to be face centered cubic with a lattice constant similar to bulk nickel. No changes in the positions of the diffraction rings were detected in the SAD patterns, and no additional diffraction rings appeared upon annealing, indicating that there is no phase transformation. The dark-field TEM images were taken by selecting a quarter of the strong diffraction rings, hence grains satisfying the selected diffraction conditions appear bright. There are notable changes in the particle size distribution from (a) to (c). To illustrate this change, the respective particle size distributions were determined as shown on the right-hand side of each image. Notice that particles smaller than $1.5 \mathrm{~nm}$ in diameter, which are a majority in the as-deposited sample [Fig. 4(a)], disappear in the sample annealed at $300{ }^{\circ} \mathrm{C}$ (b). Upon further annealing at $520^{\circ} \mathrm{C}$, the average particle size grows remarkably from 3 to $6 \mathrm{~nm}$, and the distribution becomes Gaussian-like. This is apparently caused by diffusion and is due to the higher stability of large particles.

Based on the comparison of changes in microstructures 


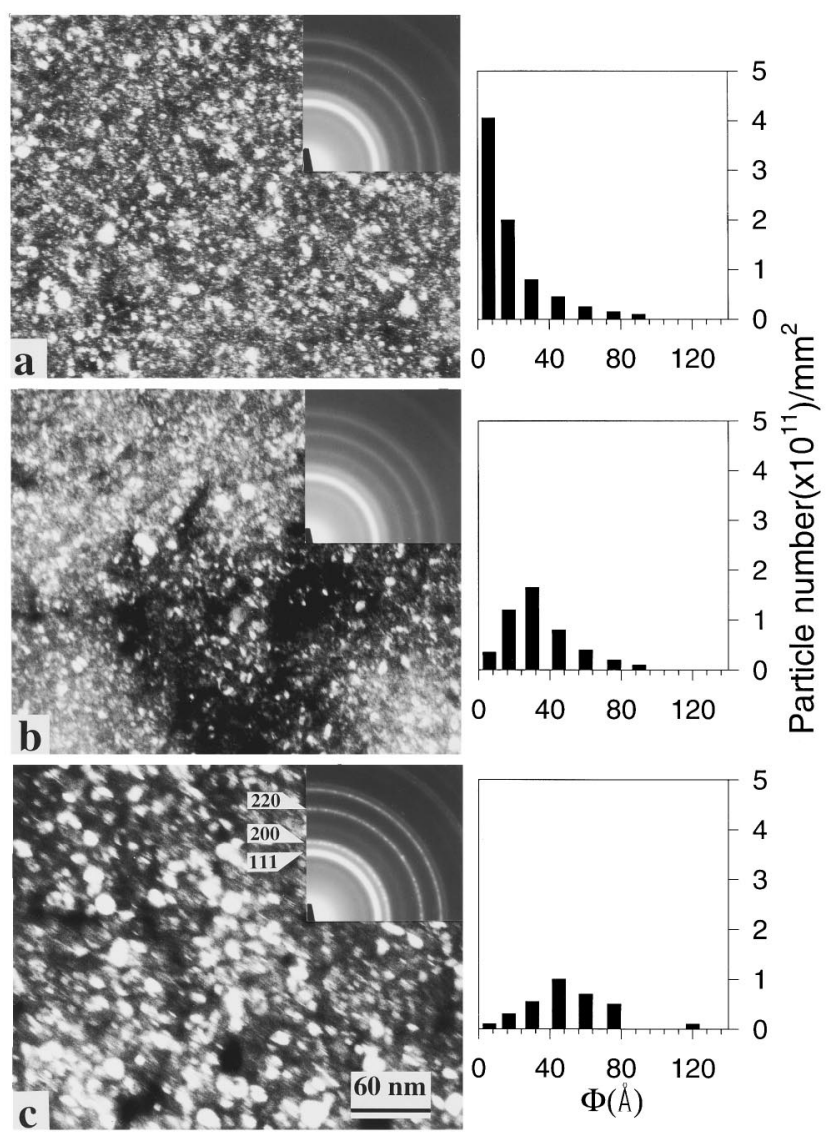

FIG. 4. Dark field TEM images for as-deposited sample (a), and those annealed at $T_{a}=300{ }^{\circ} \mathrm{C}$ (b) and at $520^{\circ} \mathrm{C}$ (c). Insets show the corresponding diffraction patterns.

with those in the transport properties, the following scenario can be suggested for the effect of annealing. The trend of altering resistivity, magnetoresistivity, and Hall resistivity, as well as the $\rho$ vs $T$ dependence, is associated with changes in particle size distribution. In the as-deposited sample, the current flows via many channels formed mainly by small granules. Upon annealing below $300{ }^{\circ} \mathrm{C}$, the smallest particles disappear, which reduces the effective number of conduction paths. That results in the increase for the resistivity, the magnetoresistivity, and the Hall resistivity (Fig. 2). This shows again that the classical percolation theory is not applicable to nanoscaled granular composites, as the classical finite-size scaling ${ }^{11}$ would predict that both the resistivity and the Hall resistivity would rather decrease with increasing granular size. At the same time, the $-\ln T$ dependent contribution to resistivity $^{12}$ is decreased, as seen in Fig. 3. Upon annealing at a higher temperature, the particles in the conduction channels start to overlap spatially, which leads to a dramatic decrease in resistivity and a change to ametallic behavior. That also results in a sharp decrease in the extraordinary Hall effect, or disappearance of the giant Hall effect. We thus conclude that the existence of the giant Hall effect is associated with the conduction mechanism that manifests itself in a $-\ln T$ dependence of resistivity in a wide temperature range up to room temperature.

We note that a $-\ln T$ dependent resistivity is very common in granular systems at the metal-insulator transition. ${ }^{13-15}$ The explanations in terms of either weak localization or electron-electron interactions both have a limited applicability, in particular at room temperature. On the other hand, the existing mechanisms of the extraordinary Hall resistivity developed for bulk metals ${ }^{9}$ are also not necessarily applicable for a percolating system at the metalinsulator transition. It is likely that a yet undetermined mechanism gives rise to both the giant Hall effect and the $-\ln T$ dependence of the resistivity in a nanoscaled granular system near the metal-insulator transition.

In summary, resistivity, magnetoresistivity, and Hall resistivity were studied for percolating granular $\mathrm{NiFe}-\mathrm{SiO}_{2}$ films annealed at different temperatures. They were all found to increase from as deposited upon annealing at $300^{\circ} \mathrm{C}$. Hall resistivity decreased by two orders of magnitude, and resistivity by almost three orders of magnitude, upon annealing at $520^{\circ} \mathrm{C}$. This pattern of resistivity and Hall resistivity change, associated with changes in particle size distribution, was accompanied by a crossover from a $-\ln T$ dependent resistivity to a metalliclike behavior. No existing theory of conduction in inhomogeneous systems close to the metalinsulator transition can be called upon to explain these correlations.

The authors would like to acknowledge Ying Fan Xu, Jie Xhie, and Stephen Leung for their assistance on film deposition and composition analysis. This work was partly supported by HKUST infrastructure Grant No. RI92/93.SC07 and partly by Hong Kong RGC Grant No. HKUST61/95P.
* On leave from Institute of Physics, Chinese Academy of Sciences, Beijing, China.

†n leave from Ioffe Physical Technical Institute, Saint Petersburg 194021, Russia.

${ }^{1}$ J. I. Gittleman, Y. Goldstein, and S. Bozowski, Phys. Rev. B 5, 3609 (1972); B. Abeles, P. Sheng, M. D. Couts, and Y. Arie, Adv. Phys. 24, 407 (1975); B. Abeles, H. L. Pinch, and J. I. Gittleman, Phys. Rev. Lett. 35, 247 (1975).

${ }^{2}$ G. Xiao and C. L. Chien, Appl. Phys. Lett. 51, 1280 (1987); A. Gavrin and C. L. Chien, J. Appl. Phys. 67, 938 (1990); C. L. Chien, ibid. 69, 5267 (1991).

${ }^{3}$ A. E. Berkowitz, J. R. Mitchell, M. J. Carey, A. P. Young, S. Zhang, F. E. Spada, F. T. Parker, A. Hutten, and G. Thomas, Phys. Rev. Lett. 68, 3745 (1992); J. Q. Xiao, J. S. Jiang, and C.
L. Chien, ibid. 68, 3749 (1992).

${ }^{4}$ G. Xiao, J. Q. Wang, and P. Xiong, Appl. Phys. Lett. 62, 420 (1993); J. Q. Wang and G. Xiao, Phys. Rev. B 49, 3982 (1994).

${ }^{5}$ A. B. Pakhomov, X. Yan, and B. Zhao, Appl. Phys. Lett. 67, 3497 (1995).

${ }^{6}$ A. B. Pakhomov, X. Yan, and Y. Xu, in Proceedings of the 40th Annual Conference on Magnets and Magnetic Materials, Philadelphia, 1995 [J. Appl. Phys. 79(8) (1996)].

${ }^{7}$ D. J. Bergman and D. Stroud, Solid State Phys. 46, 149 (1992).

${ }^{8}$ Y. Xu, B. Zhao, and X. Yan, in Proceedings of the 40th Annual Conference on Magnets and Magnetic Materials (Ref. 6).

${ }^{9}$ A. Fert and D. K. Lottis, in Concise Encyclopedia of Magnetic and Superconducting Materials, edited by J. Evetts (Pergamon, Oxford, 1992), p. 287, and references therein. 
${ }^{10}$ A. B. Pakhomov, X. Yan, B. Zhao, and Y. Xu (unpublished).

${ }^{11} \mathrm{D}$. Stauffer and A. Aharony, Introduction to the Percolation Theory, 2nd ed. (Taylor and Francis, London, 1992).

${ }^{12}$ The $\ln T$ dependence of the resistivity (Fig. 3) suggests that the conduction is unlike conventional metallic behavior. In Refs. 5,6 , and 10 , we suggested that it might be due to electronelectron interactions [B. L. Altshuler and A. G. Aronov, in Electron-Electron Interactions in Disordered Systems (NorthHolland, Amsterdam, 1985), p. 1] in the areas of granular contacts in the percolating channels, which become important when the granules are barely in physical contact. Extending that interpretation, one can suggest that upon annealing at low tempera- tures, the large particles in a small number of remaining paths grow, leading to a stronger screening of Coulomb interaction, and hence a smaller slope in the $-\ln T$ dependence as seen in Fig. 3.

${ }^{13}$ T. Chui, G. Deutscher, P. Lindenfeld, and W. L. McLean, Phys. Rev. B 23, 6172 (1981).

${ }^{14}$ N. Savvides, S. P. McAlister, C. M. Hurd, and I. Shiozaki, Solid State Commun. 42, 143 (1982).

${ }^{15}$ J. R. Beamish, B. M. Patterson, and K. M. Unruh, in Physical Phenomena in Granular Materials, edited by G. D. Cody, T. H. Geballe, and Ping Sheng (MRS, Pittsburgh, 1990), p. 129. 